PART 1 OF 3

\title{
Championing preventive care in ObGyn: A tool to evaluate for useful medical apps
}

\author{
An ACOG Presidential Task Force offers a rubric for ObGyns to apply in \\ assessing the utility and security of health apps that may aid in addressing \\ 5 areas of preventive health care
}

Hartaj K. Powell, MD, MPH, and Nicholas Kulbida, MD

\section{IN THIS ARTICLE}

App rubric

page 29

Obesity

page 31

Prepregnancy care

page 32

\section{$A C O G$}

Developed in collaboration with the American College of Obstetricians and Gynecologists

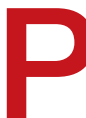

ersonalizing care is at the heart of the American College of Obstetricians and Gynecologists (ACOG) 2020-2021 President Dr. Eva Chalas' initiative to "Revisit the Visit." As obstetrician-gynecologists, we care for patients across the entirety of their life. This role gives us the opportunity to form long-term partnerships with women to address important preventive health care measures.

Dr. Chalas established a Presidential Task Force that identified 5 areas of preventive health that significantly influence the

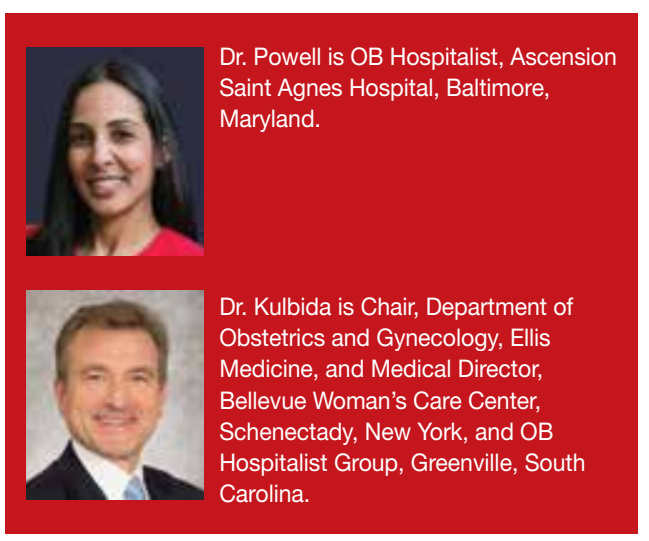

The authors report no financial relationships relevant to this article.

doi: 10.12788/obgm.0093 long-term morbidity of women: obesity, cardiovascular disease, preconception counseling, diabetes, and cancer risk. The annual visit can serve as a particularly impactful point of care to achieve specific preventive care objectives and offer mitigation strategies based on patient-specific risk factors. We are uniquely positioned to identify and initiate the conversation and subsequently manage, treat, and address these critical health areas.

\section{Harnessing modern technology}

To adopt these health topics into practice, we need improved, more effective tools both to increase productivity during the office visit and to provide more personalized care. Notably, the widespread adoption of and proliferation of mobile devices-and the medical apps accessible on them-is creating new and innovative ways to improve health and health care delivery. More than $90 \%$ of physicians use a smartphone at work, and $62 \%$ of smartphone users have used their device to gather health data. ${ }^{1}$

In addition, according to a US Food and Drug Administration (FDA) report, in 2017, 325,000 health care applications were available on smartphones; this equates to an expected 3.7 billion mobile health application downloads that year by 1.7 billion 
smartphone users worldwide. ${ }^{2}$ As of October 2020, 48,000-plus health apps were available on the iOS mobile operating system alone. ${ }^{3}$

For patients and clinicians, picking the most suitable apps can be challenging in the face of evolving clinical evidence, emerging privacy risks, functionality concerns, and the fact that apps constantly update and change. Many have relied on star rating systems and user reviews in app stores to guide their selection process despite mounting evidence that suggests that such evaluation methods are misleading, not always addressing such important parameters as usability, validity, security, and privacy. ${ }^{4,5}$

\section{Approaches for evaluating medical apps}

Many app evaluation frameworks have emerged, but none is universally accepted within the health care field.

The American Psychiatric Association's (APA) App Evaluation Model represents a comprehensive resource to consider when evaluating medical apps. It stratifies numerous variables into 5 levels that form a pyramid. In this model, background information forms the base of this pyramid and includes factors such as business model, credibility, cost, and advertising of the app. The top of the pyramid is comprised of data integration that considers data ownership and therapeutic alliance. ${ }^{6}$ Although this model is beneficial in that it provides a framework, it is not practical for point-of-care purposes as it offers no objective way to rate or score an app for quick and easy comparison.

The privately owned and operated Health On the Net (HON) Foundation is well known for its HONcode, an ethical standard for quality medical information on the internet. It uses 8 principles to certify a health website. However, the HON website itself states that it cannot guarantee the accuracy or completeness of medical information presented by a site. ${ }^{7}$ Although HON certification by a website is a sign of good intention, it is not beneficial to the practicing clinician who is looking to use an app to directly assist in clinical care.
The Agency for Healthcare Research and Quality (AHRQ) is another well-respected body that has delineated essential details to consider when using a health website. The AHRQ identifies features (similar to those of the APA pyramid and HONcode) for users to consider, such as credibility, content, design, and disclosures. ${ }^{8}$ However, this model too lacks a concise user-friendly evaluating system.

Although the FDA plans to apply some regulatory authority to the evaluation of a certain subset of high-risk mobile medical apps, it is not planning to evaluate or regulate many of the medical apps that clinicians use in daily practice. This leaves us, and our patients, to be guided by the principle of caveat emptor, or "let the buyer beware."

Thus, Dr. Chalas' Presidential Task Force carefully considered various resources to provide a useful tool that would help obstetrician-gynecologists objectively vet a medical app in practice.

\section{The Task Force's recommended rubric}

The rubric shown on page 30 for evaluating mobile drug information apps was developed by the American Society of Health-System Pharmacists (ASHP). The ASHP rubric takes into account the criteria recognized by the APA pyramid, the HON Foundation, and the AHRQ and incorporates them into a userfriendly tool and scoring system that can be applied as an evaluation checklist. ${ }^{9}$ This tool is meant to aid clinicians in evaluating medical apps, but it ultimately is the user's decision to determine if an app's deficiencies should deter its use.

While all of the criteria are relevant and important, it is incumbent on us as medical experts to pay careful attention to the accuracy, authority, objectivity, timeliness, and security of any app we consider incorporating into clinical practice. A low score on these criteria would belie any perceived usefulness or value the app may have.

When applying the rubric to evaluate the quality of an app, we should be mindful of the $\overline{\text { FAST }}$

TRACK

It is incumbent on us as medical experts to pay careful attention to the accuracy, authority, objectivity, timeliness, and security of any app we consider incorporating into clinical practice 


\section{FAST}

TRACK

There is much value in having a framework for efficiently measuring an app's benefit in clinical practice primary user and which characteristics are more important than others to effect positive changes in health. For example, in addressing obesity, it is the patient who will be interacting with the app. Therefore, it's important that the app should score, on a 1- to 4-point scale ( 1 point being major deficiencies, 4 points being no deficiencies), a 4 out of 4 on features like usefulness, functionality, and design. Coveted design features that enhance the user's experience will appeal to patients and keep them engaged and motivated. However, when addressing a woman's health with respect to cancer risk, the principal features on which the app should score 4 out of 4 would be authority, objectivity, timeliness, and accuracy.

In the upcoming articles in this series, a member of the Presidential Task Force will reference the ASHP rubric to guide clinicians in choosing apps to address one of the critical health areas with their patients. The author of the piece will highlight key features of an app to consider what would add the most value in incorporating its use in clinical practice.

It would be impossible to evaluate all health care apps even if we focused only on the medical apps relevant to obstetrics and gynecology. There is much value in having a framework for efficiently measuring an app's benefit in clinical practice. The objective of this article series is to help clinicians Revisit the Visit by providing an effective tool to evaluate a medical app.

\section{References}

1. Mobius MD website. 11 Surprising mobile health statistics. http://www.mobius.md/blog/2019/03/11-mobile-health -statistics/. Accessed January 19, 2021.

2. US Food and Drug Administration website. Device software functions including medical applications. November 5, 2019. https://www.fda.gov/medical-devices/digital-health-center -excellence/device-software-functions-including-mobile -medical-applications. Accessed March 10, 2021.

3. Statista website. Number of mHealth apps available in the Apple App Store from 1st quarter 2015 to 4th quarter 2020. https://www.statista.com/statistics/779910/health-apps -available-ios-worldwide/. Accessed January 19, 2021.

4. Campbell L. Using star ratings to choose a medical app? There's a better way. Healthline website. Updated August 3, 2018. http://healthline.com/health-news/using-ratings-to -choose-medical-app-theres-a-better-way. Accessed April 22, 2021.

5. Levine DM, Co Z, Newmark LP, et al. Design and testing of a mobile health application rating tool. NPJ Digit Med. 2020;3:74.

6. Torous JB, Chan SR, Gipson SY, et al. A hierarchical framework for evaluation and informed decision making regarding smartphone apps for clinical care. Psychiatr Serv. 2018;69:498-500.

7. Health On the Net website. The commitment to reliable health and medical information on the internet. https:// www.hon.ch/HONcode/Patients/Visitor/visitor.html. Accessed January 19, 2021.

8. Agency for Healthcare Research and Quality. Assessing the quality of internet health information. June 1999. http:// www.ahrq.gov/research/data/infoqual.html. Accessed April $22,2021$.

9. Hanrahan C, Aungst TD, Cole S. Evaluating mobile medical applications. American Society of Health-System Pharmacists eReports. https://www.ashp.org/-/media/store-files /mobile-medical-apps.ashx. Accessed January 22, 2021. 